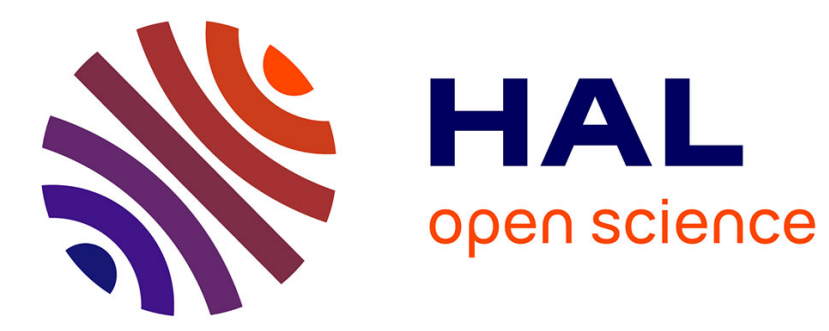

\title{
Synchronous detector as a new paradigm for automatic microembolus detection
}

Jean Marc Girault, Zheng Zhao

\section{To cite this version:}

Jean Marc Girault, Zheng Zhao. Synchronous detector as a new paradigm for automatic microembolus detection. International Journal of Biomedical Engineering and Technology (IJBET), 2014, 14 (1), pp.60. 10.1504/IJBET.2014.059059 . hal-01077276

\section{HAL Id: hal-01077276 \\ https://hal.science/hal-01077276}

Submitted on 24 Oct 2014

HAL is a multi-disciplinary open access archive for the deposit and dissemination of scientific research documents, whether they are published or not. The documents may come from teaching and research institutions in France or abroad, or from public or private research centers.
L'archive ouverte pluridisciplinaire HAL, est destinée au dépôt et à la diffusion de documents scientifiques de niveau recherche, publiés ou non, émanant des établissements d'enseignement et de recherche français ou étrangers, des laboratoires publics ou privés. 


\title{
Synchronous detector as a new paradigm for automatic microemboli detection
}

\author{
Jean-Marc Girault ${ }^{\mathrm{a}}$, Zheng Zhao ${ }^{\mathrm{a}}$ \\ ${ }^{a}$ UMR Inserm U 930, Universit Franois-Rabelais de Tours, PRES Centre-Val de Loire \\ Universit, Tours, France
}

\begin{abstract}
We propose here a new detector paradigm based on synchronous detection. Unlike standard energy detectors, this kind of detector uses the pseudo-cyclostationarity properties of blood Doppler signals. The new detector, that is synchronized with cardiac rhythm, was compared to a gold standard and a standard energy detector. Detectors were evaluated according to clinical recordings from patients with carotid stenoses. The results showed that it was possible to detect inaudible microemboli. The detection rate was improved by $30 \%$ and the false alarm rate was below $2 \%$.
\end{abstract}

Keywords: Doppler, ultrasound, microemboli, synchronous detection, cyclo-stationarity

\section{INTRODUCTION}

Early detection of cerebral microemboli is of great importance since cerebral vascular accidents represent more than two thirds of all ischemic strokes $([1])$.

Cerebral embolism is the result of migration of insoluble microparticles (pieces of fat for example) in the blood circulation. When these microemboli block an arteriol, ischemia occurs ([2]). Cerebral embolism covers a wide variety of clinical entities revealed by cerebral angiography ([5]), prosthetic heart valve insertion $([6])$, and carotid surgery $([7])$, to name but a few.

Transcranial Doppler (TCD) is currently used to detect microemboli ([3, 4]). This real time ultrasound system, also used to assess blood flow velocity

Email address: jean-marc.girault@univ-tours.fr (Jean-Marc Girault) 
for intracranial arteries, is a low-cost and non-invasive modality.

In most studies, commercial TCD detectors seem to be sufficient to detect most microembolic events ([8]). However, clinical practitioners sometimes observe previously undetected microembolic signatures that are present on the spectrogram displayed on the TCD screen during clinical examination (see Fig.1c). These undetected microemboli that are not visible on observation of the amplitude of the Doppler signal (see Fig. 1a) are sometimes unaudible. The main explanations for the inability of existing detectors to detect the smallest microemboli may be either: a threshold level adjusted by the user that is too high (see Fig. 1b) and microembolic signals of lower magnitude than that of the blood Doppler signal located at the systolic phase, i.e. the maximum amplitude level during the cardiac cycle (see Fig. 1b).

To solve the problem of inability of commercial systems to detect, a search for the implementation of reliable and robust methods was initiated. Without attempting to draw up an exhaustive list of existing methods, it should be noted that a wide variety of methods have been proposed during the last ten years such as those based on detection of sudden changes ([9]), matching pursuit ([10]), expert systems ([11]), wavelets ([12]), spectral correlation ([13]), fuzzy logic $([14,15])$, GARCH models $([16])$, and neural networks $([17])$.

The starting point of this study was based on the concept of spectral correlation ([13]) that can be viewed as a process of synchronization. Unlike the simulation studies previously presented by [13], this study evaluated the levels of performance of the synchronous detector from clinical recordings.

This new class of detector uses the fact that the nature of the microembolic Doppler signal is different from that of the blood Doppler signal. Here we contrast the cyclic nature of the blood Doppler signal to the unpredictable nature (i.e. uncyclic) of the Doppler signal of microemboli.

Our new class of detector was compared to the most frequently used commercial detectors, i.e. non-synchronized energy detectors, to demonstrate its relevance.

\section{STANDARD METHOD}

The energy of the Doppler signal seems to provide the best information from which the presence of microemboli can be detected. This can mainly be explained by the fact that the energy of the backscattered Doppler signal is proportional to the size of the microembolus to the power of 6 while the 

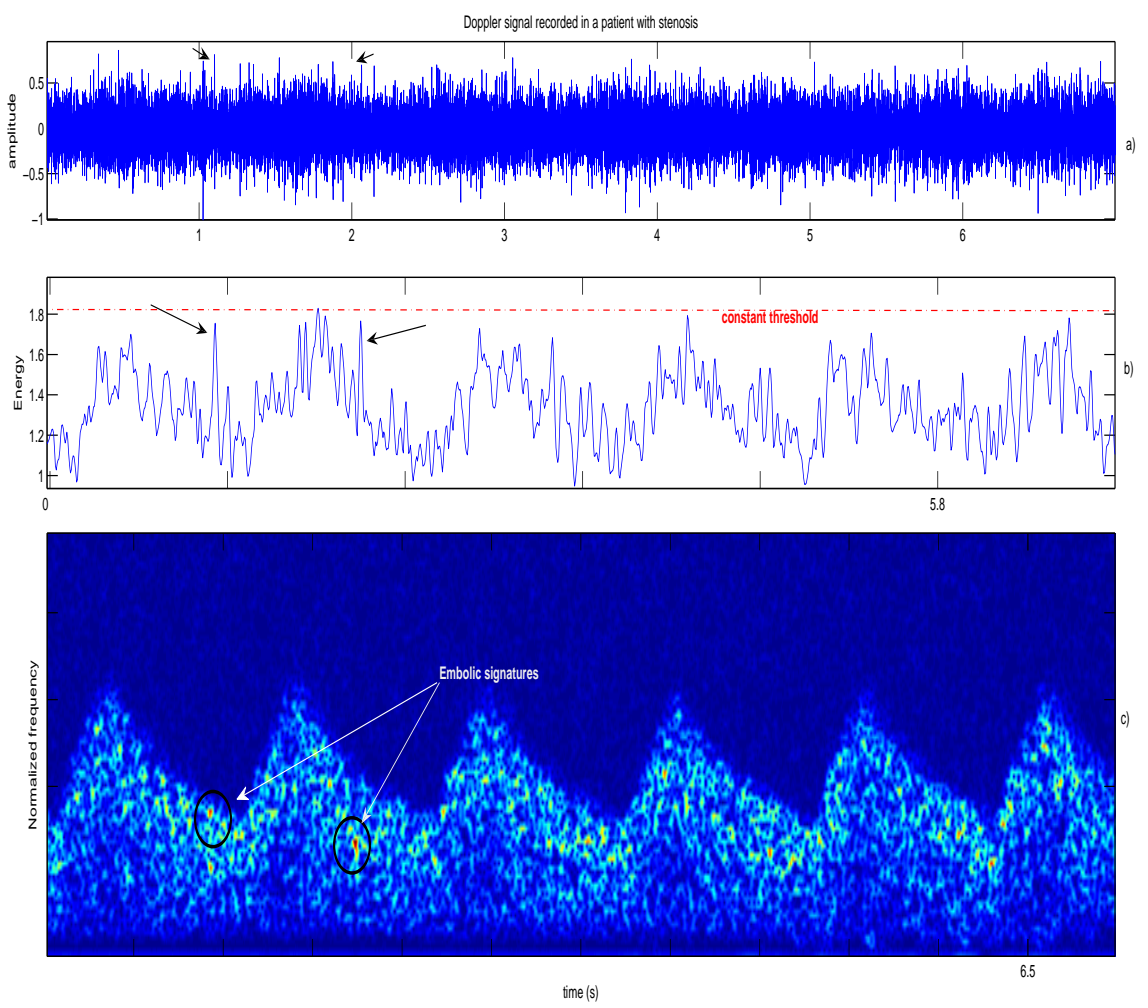

Figure 1: Doppler signal with two inaudible microemboli recorded in a patient with stenosis. (top) Time representation of the Doppler signal. (middle) Instantaneous energy of the Doppler signal. (bottom) Spectrogram of the Doppler signal.

Rayleigh scattering holds, in other words larger the microembolus, the higher the intensity of the Doppler signal.

Two kinds of energy detector can be used, those based on time estimators and those based on spectral estimators. As spectral estimators are presented in the next section, only the time estimator is presented in this section. The time estimator of the instantaneous energy can be obtained by:

$$
\left.E(t)=\int_{-\infty}^{+\infty} \mid \int_{-\infty}^{+\infty} x(\tau) g^{*}(t-\tau)\right)\left.d \tau\right|^{2} d t
$$

where $g(t)$ is a Hanning sliding window and $*$ stands for the conjugation operation. 
As previously explained, in commercial devices the decision information (DI) on which detection is based is currently the instantaneous energy $E(t)$. This DI is then compared to a constant threshold. Two strategies are then possible:

- the constant threshold is fixed empirically by the user for the entire examination. This arbitrary threshold is patient-, operator- and devicedependent. As this threshold level is not the same at each examination, comparison is difficult;

- the constant threshold is set automatically from the instantaneous energy. This threshold is often evaluated as a mean value derived from previous energy over a few seconds. This threshold is thus a statistical threshold.

Whatever the method, DI is then compared to a non-time-varying threshold $\lambda$. If the decision information $D I=E(t)$ is greater than the threshold $\lambda$ then an embolus is considered to be detected (hypothesis $H_{1}$ ), otherwise no embolus is detected (hypothesis $H_{0}$ ). This can be expressed as follows:

$$
E(t) \stackrel{H_{1}}{ } \underset{ }{\gtrless} \lambda .
$$

In this study only statistical thresholds were taken into consideration. This means that a training period was required to extract statistics. After observing that the statistics of the instantaneous energy followed gaussian law, the following threshold $\lambda$ was proposed:

$$
\lambda=\mu+\alpha \sigma
$$

where $\alpha$ was a parameter to be adjusted, and $\mu$ and $\sigma$ were the mean value and the standard deviation of the instantaneous energy, respectively.

From our experience, a training period of 5 seconds and $\alpha$ ranging from 3 to 5 seemed to be optimal values.

\section{NEW METHOD}

The new type of detector that we propose is based on the use of a detector synchronized with the cardiac rhythm. The solution that we proposed consisted of four parts (Fig.2): 


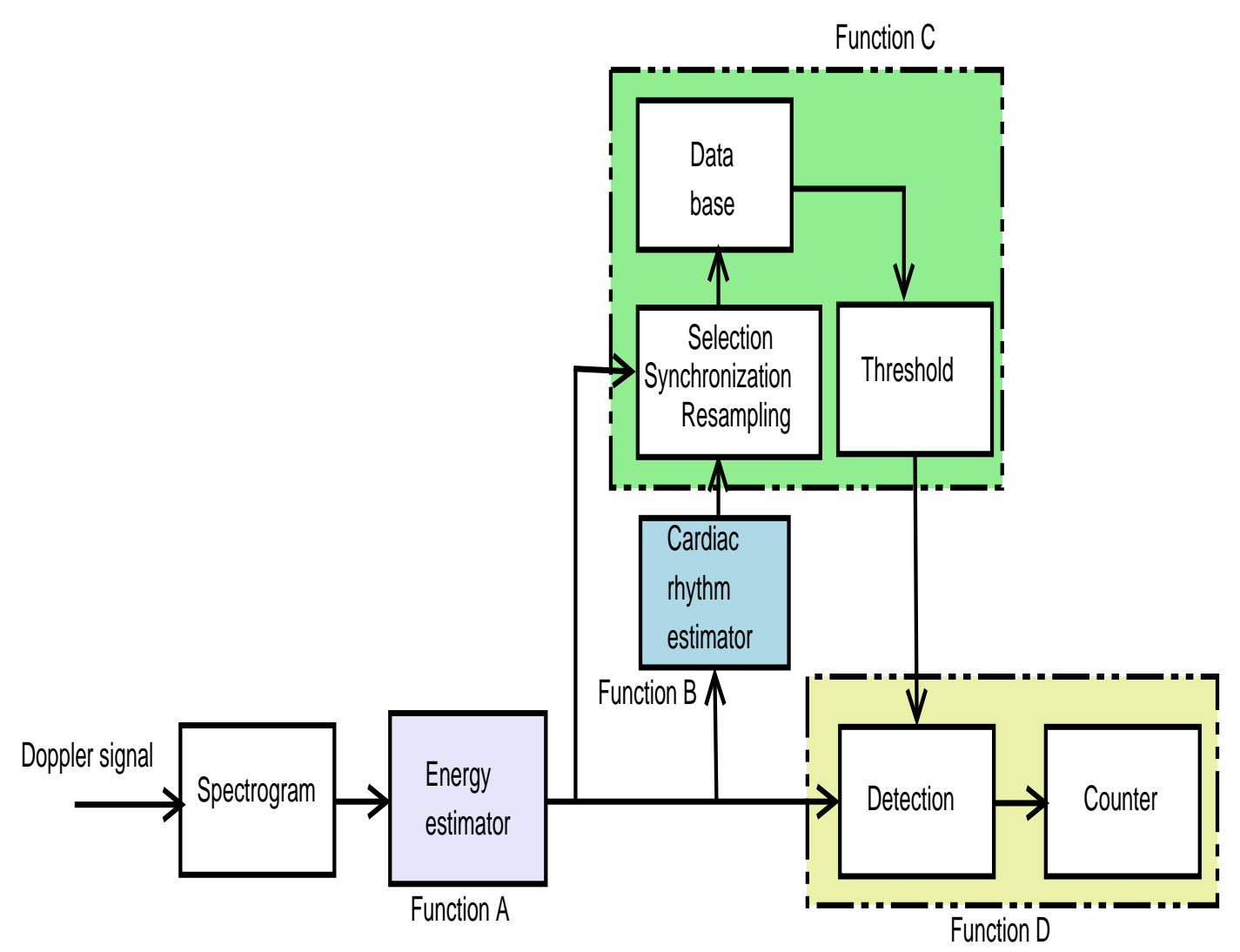

Figure 2: Function diagram of the new system detector without artefact rejection.

1. Evaluation of the instantaneous energy of the Doppler signal (function A in Fig.2);

2. Evaluation of the cardiac rhythm and identification of each time index representing the beginning of each cardiac cycle (function B in Fig.2);

3. Evaluation of the cardiac statistical threshold by extracting the Doppler energy signal of each cardiac rhythm from previous time indices, and by resampling each Doppler energy signal in order to provide the same length (function C in Fig.2);

4. Detection and enumeration of events outside statistics (function D in Fig.2).

Note that for standard commercial detectors introduced in the previous section, there is no step 2, and step 3 may or may not be present. 


\subsection{Energy estimator}

Several estimators can be used to assess the instantaneous energy of Doppler signals. Here we focus on the spectral estimator since it is already included in the spectrogram calculation of commercial TCD systems (function A in Fig.2).

The instantaneous energy is obtained from the spectrogram by:

$$
\left.S_{x}(t, f)=\mid \int_{-\infty}^{+\infty} x(\tau) g^{*}(t-\tau)\right)\left.e^{-j 2 \pi f \tau} d \tau\right|^{2},
$$

where $g(t)$ is a Hanning sliding window and $*$ stands for conjugation operation.

An illustration of the spectrogram of a recorded Doppler signal is presented in Fig. 1c. The instantaneous energy over the entire spectrum for a fixed time $t$, can be obtained by:

$$
E(t)=\int_{-\infty}^{+\infty} S_{x}(t, f) d f
$$

An illustration of the instantaneous energy of a Doppler signal with microemboli is presented in Fig.1b.

As expected, the instantaneous energy fluctuated considerably since it revealed the stochastic nature of billions of red blood cells randomly located in the artery. Furthermore, repetitive patterns for each cardiac cycle can be seen. The pattern shape describes each part of the cardiac cycle, such as the systolic and the diastolic phases.

\subsection{Cardiac rhythm estimator}

The instantaneous cardiac rhythm can be obtained using different modalities (ultrasound and EKG) and different estimators based on the instantaneous frequency or on the instantaneous energy of the Doppler signal, estimators being temporal or spectral.

Many temporal algorithms based on the correlation function of the Doppler signals have been proposed to evaluate the fetal heart rate (e.g. [18]). However, as the instantaneous energy had already been evaluated in the previous steps, it seemed preferable to use the instantaneous energy to evaluate the cardiac rhythm (function B in Fig.2).

To determine the cardiac rhythm from the instantaneous energy $E(t)$, we proposed extracting the fundamental component $E_{0}(t)$ of the instantaneous 
energy. This fundamental component was extracted from the spectrogram $S_{x}(t, f)$ by using a low pass filter :

$$
S_{0}(f, t)=S_{x}(f, t) G(f, t),
$$

where $G(f, t)$ is the square modulus of the time-varying transfer function of the low pass filter. This latter transfer function can be simply a rectangular function centered around the null frequency:

$$
G(f, t)=G(f)=\operatorname{Rect}_{F_{c}}(f),
$$

where $F_{c}$ is the cut-off frequency of the low pass filter. By using the inverse Fourier transform and an appropriate value of $F_{c}$, the filtered energy signal is written simply as:

$$
E_{0}(t)=A_{0} \cos \left(2 \pi f_{0} t\right),
$$

where $f_{0}$ corresponds to the cardiac frequency. This kind of filter extracted the fundamental component $f_{0}$ of the Fourier series of the cardiac rhythm.

By detecting the maxima of the fundamental component of the instantaneous energy $E_{0}(t)$, it was possible to index each cardiac cycle. Note that it would also be possible to index each cardiac rhythm by detecting the minima or the zero-crossings.

As an illustration, we report on Fig.3 the histograms of normalized cardiac rhythms obtained from two different recordings. The first histogram with a gaussian shape had a relatively smaller variance compared to the second histogram. The difference was due to the large number of ventricular extra-systoles in the second recording. This could be problematic, since our synchronous detector was based on the regularity of the cardiac rhythm. To overcome this problem, all cardiac cycles that exceeded $20 \%$ of the mean value of the normalized cardiac rhythm were removed. Derived from the analysis of our database, this value seemed to be the best trade-off between i) keeping enough cardiac cycles to maintain the rate of non-detection of microemboli at its lowest and ii) removing sufficient outlier cardiac cycles so that the synchronization process was not too disturbed.

\subsection{Statistical threshold}

This step was probably the most crucial. It consisted of determining the cardiac statistical threshold by extracting each Doppler energy signal from previous time indices (function $\mathrm{C}$ in Fig.2). However, as the cardiac rhythm 

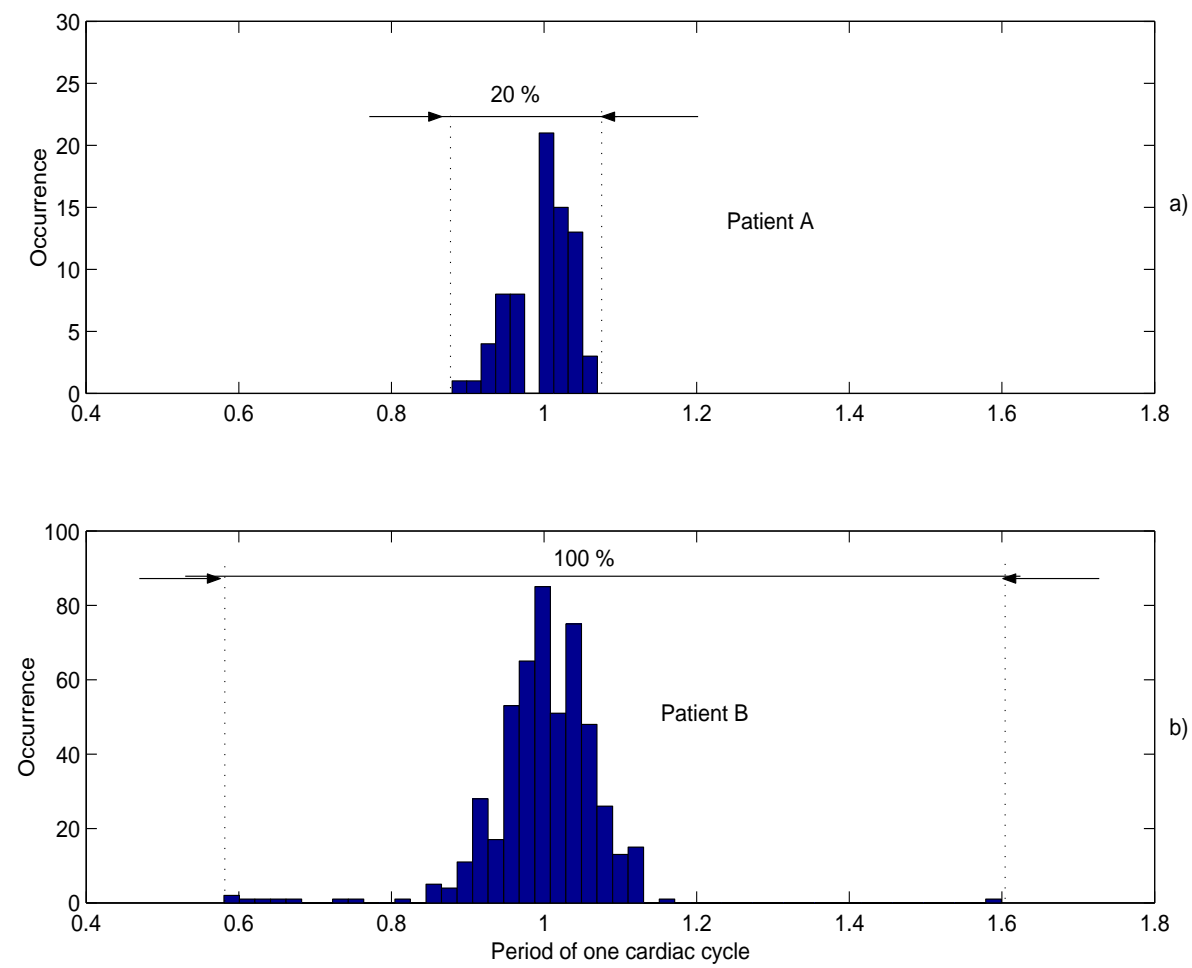

Figure 3: Histograms of normalized cardiac rhythms obtained from two different recordings. a) Narrow gaussian histogram obtained for one patient. b) Wide gaussian histogram obtained for another patient.

can fluctuate slightly, each extracted energy signal was resampled in order to give them all the same length. For a gaussian statistic, the statistical threshold can be evaluated as follows:

$$
\lambda(t)=\mu(t)+\alpha \sigma(t),
$$

where $\alpha$ is a parameter to be adjusted, and $\mu(t)$ and $\sigma(t)$ the mean value and the standard deviation of the energy signal, respectively.

From study of our data set it seemed best to set $\alpha$ ranging from 3 to 5. Note that to maintain a certain degree of adaptativity, accuracy and robustness in our approach, a sufficient duration to determine the statistical threshold was required. It was obvious that the longer the duration of the 

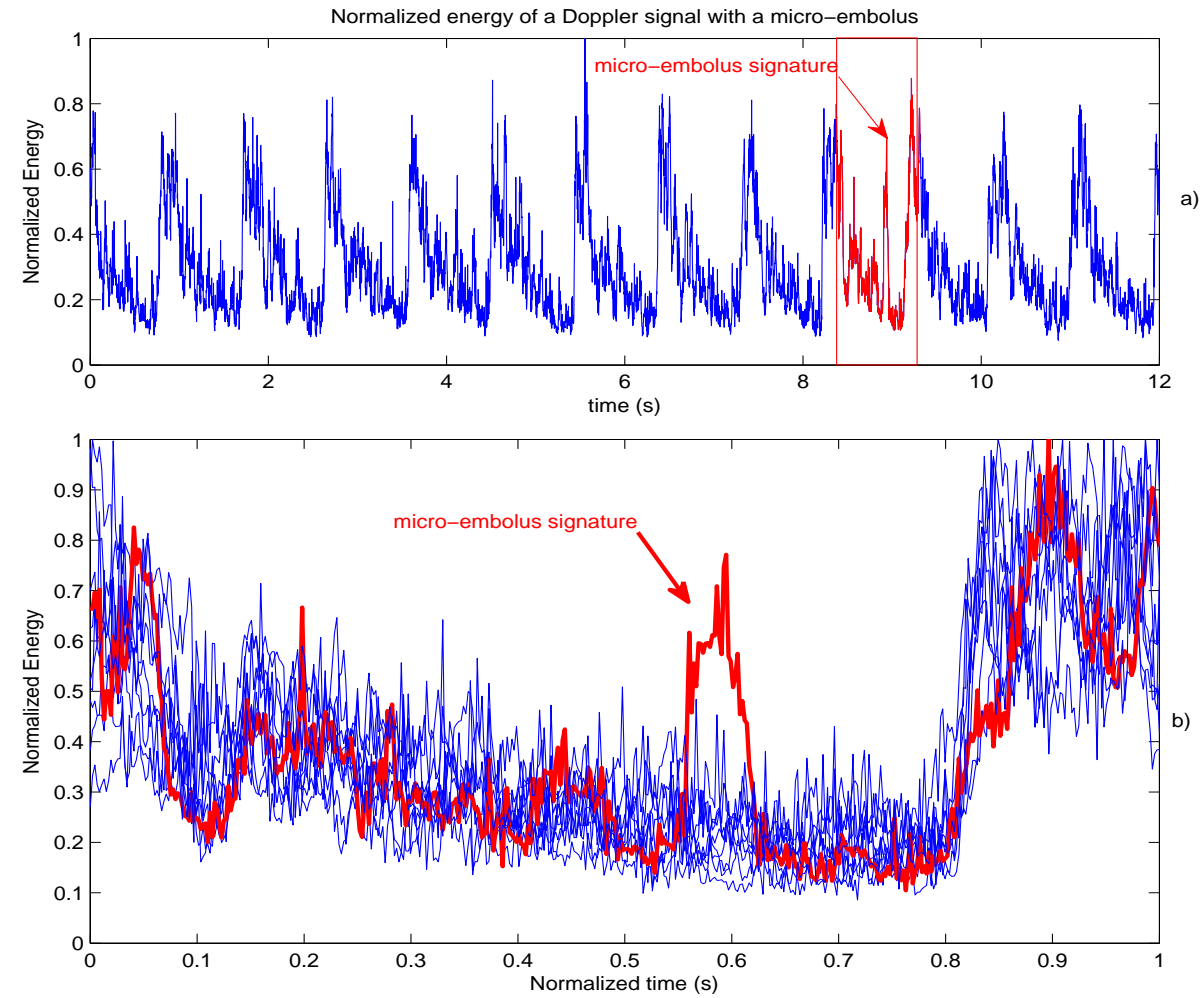

Figure 4: Microembolus signature superimposed on the Doppler energy. (a) Doppler energy signal of 12 seconds estimated from our data set. (b) After indexing and resampling of each energy signal, energy signals were superimposed on each other. Each energy signal had a normalized duration.

training phase, the more accurate the detection. On the other hand, the shorter the duration, the easier it is for the system to adapt to the presence of artefacts or any other changes. Our experience indicated that a duration of 5 seconds was a good trade-off to evaluate the statistical threshold. Note that the threshold was updated for each cardiac cycle. This involved an overlap of four cardiac cycles since the duration was of five cardiac cycles.

An illustration is given in Fig. 4 where the instantaneous energy was evaluated over 12 seconds. A high intensity transient signal was observed in the tenth cardiac cycle. After indexing and resampling, energy signals $E_{i}(t)$ of normalized duration were superimposed on each other. $i$ represents the index of the cardiac cycle. The tenth cardiac cycle is superimposed in red 
in both Fig.4a and Fig.4b. The microembolus signature is highlighted in Fig.4b. This type of example explains why our detector has the potential to detect the smallest microemboli even though their energy signatures were below the systolic energy.

\subsection{Detection and enumeration}

In addition to the other previously introduced strategies, a third strategy consisting of determining a time-varying threshold during the cardiac cycle can be proposed. Detection is said to be "synchronized" if comparison of the energy $E$ with the threshold $\lambda$ is performed at the same time position $t_{0}$ in the normalized cardiac cycle $T$, i.e. periodically for all $t=t_{0}+k T$. For a time position $t_{0}$ in a normalized cardiac cycle and the normalized cardiac duration $T=1$, synchronous detection consists of comparing an energy value $E\left(t_{0}\right)$ at time $t_{0}$, to a threshold $\lambda\left(t_{0}\right)$. This statistical threshold, set for example at $\lambda\left(t_{0}\right)=\mu\left(t_{0}\right)+5 \sigma\left(t_{0}\right)$, is therefore obtained from a preliminary setting. $\mu\left(t_{0}\right)$ and $\sigma\left(t_{0}\right)$ are the mean and the standard deviation taken at time position $t_{0}$ of the energy cycle, respectively. For example, $\mu\left(t_{0}\right)$ is obtained by adding the energy for each time value $t=t_{0}+k T$ as described below:

$$
\mu\left(t_{0}\right)=\frac{1}{N} \sum_{k=0}^{N-1} E\left(t_{0}+k T\right) .
$$

A setting phase (of a few seconds) is therefore necessary during the examination to evaluate statistics. Statistics are updated periodically thereafter during the examination. Note that this synchronized detection is equivalent to detection based on an adaptive threshold $\lambda\left(t_{0}\right)$, this adaptive threshold itself being based on the statistics of the signal being analyzed:

$$
E(t, T) \stackrel{H_{1}}{\gtrless} \lambda(t, T) .
$$

As the comparison is performed periodically with the normalized cardiac period $T, E(t)$ and $\lambda(t)$ become $E(t, T)$ and $\lambda(t, T)$. This latter point is very important, because now the threshold is no longer obtained from an empirical study but rather from a statistical study of a non-stationary process. 


\begin{tabular}{|c|c|c|c|}
\hline \hline Parameters & $\begin{array}{c}\text { Gold } \\
\text { standard }\end{array}$ & $\begin{array}{c}\text { Standard } \\
\text { detector }\end{array}$ & $\begin{array}{c}\text { Synchronous } \\
\text { detector }\end{array}$ \\
\hline \hline$N$ & 4.2 & 3.1 & 5.5 \\
\hline$P c t$ & $100 \%$ & $75 \%$ & $130 \%$ \\
\hline
\end{tabular}

Table 1: (First line) Mean number $\bar{N}$ of microemboli detected from clinical recordings of 30 minutes. (Second line) Percentage of detection Pct comparison to the Gold standard. Around $8 \%$ of cardiac cycles were unusable.

\section{RESULTS AND DISCUSSION}

\subsection{Protocol}

The new kind of detector proposed here was tested on 20 clinical recordings. These 30-minute recordings were carried out in the Ultrasound Department of Bretonneau Hospital in Tours, France. The waky-TCD ${ }^{T M}$ of Atys Medical was used. A $2 \mathrm{MHz}$ ultrasound probe was used to explore the middle cerebral artery. The pulse repetition frequency was mostly set at 6.4 $\mathrm{kHz}$ and the US power did not exceed $150 \mathrm{~mW} / \mathrm{cm}^{2}$. Volunteer patients with carotid stenoses took part in the measurement protocol which was approved by the Clinical Investigation Center CIC-IT 806 CHRU of Tours.

The gold standard corresponded to visual and audible detection of microemboli by both non-hospital practitioners and hospital practitioners. The automatic thresholds were set in order to guarantee the detection of all microemboli obtained with the gold standard.

\subsection{Results}

The first interesting findings showed that the false alarm rate for all automatic detectors was less than $2 \%$. The second important result, shown in Table1, demonstrated that about $25 \%$ of microemboli were not detected with the automatic standard detector compared to the gold standard. The third interesting result was that the synchronous detector counted about $30 \%$ of detections more than the gold standard and about $75 \%$ of detections more than the standard energy detector. Note that such results were obtained from $92 \%$ of the whole recording. Indeed, $8 \%$ of cardiac cycles were unusable according to our validity criterion for cardiac cycles. This means that $8 \%$ of cardiac cycles were outside the authorized range of $20 \%$ around the mean cardiac rhythm. Most of these unusable cardiac rhythms corresponded 
to ventricular extra-systoles. The percentage of unusable cardiac rhythms reached $37 \%$ for some patients and sometimes $1 \%$. This means that the quasi-cyclostationarity hypothesis was validated on the whole, but prudence is required.

In order to be sure that the $30 \%$ of over-detections were true microemboli undetected by the gold standard procedure, recordings were listened to again at a playing speed reduced by a factor of two and visualized twice on the spectrogram. One spectacular case is reported in Fig. 1.

\subsection{Discussion}

The results summarized in Table 1 confirmed that the standard detector was not a good automatic detector since it missed $35 \%$ of microemboli detected by visualizing the spectrogram on the TCD screen and by listening to audible signatures of microemboli. However, these levels of performance were not too bad since detection was performed automatically, i.e. without operator-dependent pre-setting. The levels of performance of our new kind of detector were therefore very encouraging since it made it possible to detect inaudible microemboli. The results confirmed that the human auditory system is not perfect. However, although our new type of detector surpassed the standard detector, these good results must be confirmed on a larger dataset.

One short term prospect for this work would be to improve further the levels of performance of the automatic detectors tested. Several approaches might be explored, the first being proposing other values for the training and updating periods, and the second proposing other values of $\alpha$. These two approaches seem to be relevant since their are patient-dependent.

Finally, note that no microemboli were detected with the gold standard or the standard energy detector in the unusable cardiac rhythms. However, there is no guarantee that microemboli might not sometimes appear in unusable cardiac cycles. There is currently no solution to this possibility and this will be the objective of further research.

\section{CONCLUSION}

Automatic detectors that were not operator-dependent were tested in this study. The levels of performance of the new proposed detector surpassed those of the gold standard and the standard detector. However, although the synchronous detector demonstrated the best levels of performance, it is clear that this detector based on pseudo-cyclo-stationary properties might 
sometimes fail for certain patients with high levels of variations in cardiac rhythm. Further quantitative study should be planned to disprove or confirmed this possibility.

\section{References}

[1] V. L. Feigin. (2005) 'Stroke Epidemiology in the Developing World', The Lancet, Vol. 365, pp. 2160-2161.

[2] M. Siebler,A. Nachtmann,M. Sitzer,G. Rose,A. Kleinschmidt,J. Rademacher, H. Steinmetz. (1995) 'Cerebral Microembolism and the Risk of Ischemia in Asymptomatic High-Grade Internal Carotid Artery Stenosis', Stroke, Vol. 26, pp. 2184-2186.

[3] R. Aaslid, T.M. Markwalder, H. Nornes (1982) 'Non invasive transcranial Doppler ultrasound recording of flow velocity in basal cerebral arteries', J. Neurosurg., Vol. 57 (6), pp. 769?774.

[4] M.P. Spencer, G.I. Thomas, S.C. Nicholls and L.R. Sauvage. (1995) 'Detection of middle cerebral artery emboli during carotid endarterectomy using transcranial Doppler ultrasonography', Stroke, Vol. 21, pp. 415423.

[5] H.S. Markus, A. Loh, D. Israel, T. Buckenham, A. Clifton, M.M. Brown, (1993) 'Microscopic air embolism during cerebral angiography and strategies for its avoidance', The Lancet, Vol. 341, pp. $784 ? 787$.

[6] D. Georgiadis, D. Grosset, A. Kelman, A. Faichney, K.R. Lees. (1994) 'Prevalence and characteristics of intracranial microemboli signals in patients with different types of prosthetic cardiac valves', Stroke, Vol. 25 (11), pp. 587?592.

[7] R.G.A. Ackerstaff, C. Jansen, F.L. Moll. (1996) 'Carotid endarterectomy and intraoperative emboli detection', Echocardiography, Vol. 13 (5), pp. $543 ? 550$.

[8] R. Aaslid. (1992) 'Transcranial Doppler, chapter : Developments and principles of transcranial Doppler', Edited by D.W. Newell and $R$. Aaslid, Raven Press, Ltd, New-York. 
[9] J.M. Girault, D. Kouamé, A. Ouahabi and F.Patat. (2000) 'Microembolic detection: an ultrasound Doppler signal processing view point', Ieee Trans. Biomed. Eng., Vol. 47, pp. 1431-1439.

[10] G. Devuyst, J.-M. Vesin, P.-A. Despland, and J. Bogousslavsky (2000) 'The matching pursuit: A new method of characterizing microembolic signals?', Ultrasound Med. Biol., Vol. 26, pp 1051?1056.

[11] Fan L, Evans D., Naylor A. (2001) 'Automated embolus identification using a rule based expert system', Ultrasound Med. Biol. , Vol. 27, pp $1065 ? 1077$.

[12] N. Aydin, F. Marvasti, H.S. Markus (2004) 'The use of the wavelet transform to describe embolic signals', IEEE Trans. Info. Techn. Biomed., Vol. 8 (2), pp 182-190.

[13] J.M. Girault, M. Biard, D. Kouamé, A. Bleuzen, and F. Tranquart. (2006) 'Spectral Correlation of the embolic blood Doppler signal', ICASSP, Toulouse, pp. 2448-2451.

[14] D. Kouamé, M. Biard, J.-M. Girault, A. Bleuzen (2006) 'Adaptive AR and Neurofuzzy Approaches: Access to Cerebral Particle Signatures', IEEE Trans. Info. Techn. Biomed., Vol. 10 (3), pp 559-566.

[15] D. Xu, Y. Wang (2007) 'An automated feature extraction and emboli detection system based on the PCA and fuzzy sets', Computers in Biology and Medicine, Vol. 37, pp 861-871.

[16] J.-M. Girault, S. Ménigot, L. Drebine, (2010) 'Automatic Detection of microemboli by means of a generalized autoregressive conditional heteroskedasticity model', Jasa, Vol 128 (4) , pp 2304-2305

[17] N. Benoudjit, K. Ferroudji, M. Bahaz, A. Bouakaz (2011) 'In vitro microemboli classification using neural network models and RF signals', Ultrasonics, vol 51, pp. 247-252.

[18] I. Voicu, J.M. Girault, M. Fournier-Massignan, D. Kouamé. (2010) 'Robust Estimation of Fetal Heart Rate from US Doppler Signals', International Conference on Ultrasonics, Santiago, Physics Procedia, vol 3, pp. 691-699. 